\title{
Traitements de la cholestase chronique chez l'enfant
}

\author{
Fernando Alvarez \\ CHU Sainte-Justine, Université de Montréal, Montréal, Qué., Canada
}

\section{Mots-clés}

Cholestase chronique - Acide ursodésoxycholique •

Rifampicine - Résines échangeuses d'ions - Acides gras $\omega-3$ • $\mathrm{N}$-acétylcystéine $\cdot$ Antagoniste des opiacés

\section{Résumé}

Peu de traitements spécifiques de la cholestase chronique de l'enfant sont disponibles. La plupart des stratégies thérapeutiques réduisent la rétention des composants de la bile ou pallient certaines des conséquences d'une cholestase chronique. L'acide ursodésoxycholique est l'agent pharmacologique le plus souvent administré chez l'enfant atteint de cholestase chronique. Cet acide biliaire est administré à la posologie de 10 à $30 \mathrm{mg} / \mathrm{kg} /$ jour chez les patients présentant une mucoviscidose, des erreurs innées du métabolisme des acides biliaires, une cholestase intrahépatique familiale progressive, une cholangite sclérosante, une atrésie biliaire, un syndrome d'Alagille ou recevant une nutrition parentérale totale. L'acide ursodésoxycholique augmente principalement le débit biliaire et exerce un effet stabilisateur de membrane, ce qui réduit la toxicité des acides biliaires plus hydrophobes. La rifampicine, un antibiotique, à la posologie de 10 à 20 mg/kg/jour est très efficace pour le soulagement du prurit. Des effets similaires sont obtenus au moyen de résines échangeuses d'ions non absorbables. De plus, ces molécules diminuent la cholestérolémie, ce qui contribue à réduire les xanthomes. La compensation de certaines carences créées par une nutrition parentérale totale par l'administration d'acides gras essentiels ou de cystéine peut prévenir les troubles hépatiques associés ou contribuer à leur amélio- ration. Dans certaines affections cholestatiques, des interventions chirurgicales peuvent faciliter la suppression de l'obstacle à l'écoulement de la bile, comme par exemple la portoentérostomie chez les patients présentant une atrésie des voies biliaires En cas de cholestase intrahépatique, une amélioration clinique et biochimique peut être enregistrée après une dérivation de la bile ou une autre intervention (exclusion de l'iléon) limitant l'absorption des acides biliaires par l'intestin. Dans le futur, l'association de ces différents agents pharmacologiques, accroissement du débit biliaire, protection des membranes cellulaires ou correction de carences nutritionnelles devrait contribuer à une amélioration de la qualité de vie des enfants atteints de cholestase chronique et, par la suite, retarder la nécessité d'un traitement plus drastique comme une transplantation hépatique. Des progrès de la thérapie génique et de la transplantation d'hépatocytes pourraient être également très utiles. Cependant, de nombreuses années d'intenses recherches sont encore nécessaires avant qu'une étude, même pilote, de l'une de ces thérapies puisse être envisagée dans les hépatopathies résponsables d'une cholestase chronique.

Copyright $\odot 2009$ Nestec Ltd., Vevey/S. Karger AG, Basel

Une cholestase chronique chez l'enfant est la conséquence de causes très diverses dont très peu relèvent d'un traitement spécifique. Dans la plupart des cas, des traitements médicaux ou chirurgicaux ont été développés afin de prévenir ou de traiter des complications secondaires à une rétention des composants de la bile. Les traitements existants sont principalement de soutien, tentant de ré-

\section{KARGER}

Fax +4161306 1234

E-Mail karger@karger.ch

www.karger.com
(C) 2009 Nestec Ltd., Vevey/S. Karger AG, Basel 0250-9644/08/0663-0127\$24.50/0

Accessible en ligne à:

www.karger.com/anf
Prof. Fernando Alvarez, MD

Division of Gastroenterology, Hepatology and Nutrition

CHU Sainte-Justine, University of Montreal

3175, Cote Sainte-Catherine road, Montreal, Qué. H3T 1C5 (Canada)

E-Mail fernando.alvarez@umontreal.ca 
Tableau 1. Traitements disponibles de la cholestase chronique chez l'enfant

\begin{tabular}{|c|c|c|c|c|}
\hline Traitement & Indication & Contre-indication & Effets indésirables & Posologie \\
\hline AUDC & $\begin{array}{l}\text { Influence la pathogenie } \\
\text { de CIFP, mucoviscidose, } \\
\text { NPT; «prurit» }\end{array}$ & $\begin{array}{l}\text { Obstruction des voies biliaires } \\
\text { (par exemple échec d'une } \\
\text { portoentérostomie pour AVB) }\end{array}$ & Diarrhée & $20-30 \mathrm{mg} / \mathrm{kg} /$ jour \\
\hline Rifampicine & «Prurit» & Infection à mycobactéries & & $10-20 \mathrm{mg} / \mathrm{kg} /$ jour \\
\hline $\begin{array}{l}\text { Résines } \\
\text { échangeuses } \\
\text { d'ions non } \\
\text { absorbables }\end{array}$ & $\begin{array}{l}\text { «Prurit» } \\
\text { Hyper- } \\
\text { cholestérolémie }\end{array}$ & $\begin{array}{l}\text { Sténose ou sub-occlusion } \\
\text { intestinale }\end{array}$ & $\begin{array}{l}\text { Constipation } \\
\text { Acidose hyper- } \\
\text { chlorémique }\end{array}$ & $\begin{array}{l}8-16 \mathrm{~g} / \text { jour par voie orale } \\
\text { en } 2-3 \text { prises (augmentation } \\
\text { progressive à partir de } 2 \mathrm{~g} / \\
\text { jour par voie orale) }\end{array}$ \\
\hline $\begin{array}{l}\text { Antagonistes } \\
\text { des opiacés }\end{array}$ & «Prurit» & - & Réactions de sevrage & $?$ \\
\hline Acides gras $\omega-3$ & $\begin{array}{l}\text { Hépatopathie induite } \\
\text { par NPT }\end{array}$ & - & - & $\begin{array}{l}1 \mathrm{~g} / \mathrm{kg} / \text { jour (augmentation } \\
\text { progressive à partir de } 0,2 \\
\mathrm{mg} / \mathrm{kg} / \text { jour) }\end{array}$ \\
\hline NAC & $\begin{array}{l}\text { Hépatopathie induite } \\
\text { par NPT }\end{array}$ & - & - & $\begin{array}{l}70-135 \mathrm{mg} / \mathrm{kg} / \text { jour (aug- } \\
\text { mentation progressive à } \\
\text { partir de } 20 \mathrm{mg} / \mathrm{kg} / \text { jour) }\end{array}$ \\
\hline
\end{tabular}

Des associations de certains de ces médicaments sont habituellement nécessaires. AVB = atrésie des voies biliaires.

duire l'accumulation des acides biliaires dans l'organisme et ses conséquences: fibrose hépatique progressive, prurit, hyperlipidémie et asthénie.

Une réduction de la quantité d'acides biliaires dans la lumière de l'intestin est responsable d'une malabsorption des lipides. Une stéatorrhée peut devenir une cause de malnutrition et de carence en vitamines liposolubles dans la cholestase chronique. Certains traitements décrits plus bas, visant à éviter la recirculation entérohépatique des acides biliaires, peuvent aggraver cette situation.

Des agents pharmacologiques tels l'acide ursodésoxycholique (AUDC) et la rifampicine améliorent la cholérèse en accroissant l'excrétion des acides biliaires par les hépatocytes. Les résines échangeuses d'ions non absorbables fixent les acides biliaires dans la lumière de l'intestin, diminuant leur circulation entérohépatique. Tous ces agents accroissent la cholérèse et diminuent le prurit et la cholestérolémie. Les antagonistes des opiacés ont été proposés pour le traitement du prurit mais leur disponibilité limitée ainsi que leurs effets indésirables ont restreint leur utilisation en pédiatrie (tableau 1).

Des interventions chirurgicales peuvent être curatives, par exemple la méthode de Kasai chez certains patients atteints d'atrésie biliaire, ou palliatives, par exemple la dérivation biliaire partielle et l'exclusion de l'iléon chez des patients atteints de syndrome d'Alagille ou de cholestase intrahépatique familiale progressive (CIFP). Cette dernière intervention pourrait améliorer le prurit, diminuer la rétention de cholestérol et éviter une évolution vers une cirrhose.

\section{Acide ursodésoxycholique}

L'AUDC (acide $3 \alpha, 7 \beta$-dihydroxy-5 $\beta$-cholane-24-oїque) est présent en petites quantités dans la bile humaine (jusqu'à $3 \%$ ) et résulte de l'épimérisation en $7 \beta$ de l'acide chénodésoxycholique par des bactéries coliques [1].

Le groupe hydroxy $\beta$, et non $\alpha$, en position 7 donne à l'AUDC une hydrophilie plus élevée que celle de l'acide chénodésoxycholique, son précurseur. Ainsi, en raison de sa moindre hydrophobie, l'AUDC est médiocre pour la formation de micelles. De plus, il est peu absorbé dans l'intestin proximal [1].

\section{Mécanismes d'action}

- Remplacement des acides plus hydrophobes dans le pool circulant et au niveau des membranes cellulaires. Au bout de 6 mois de traitement à $10-12 \mathrm{mg} / \mathrm{kg} / \mathrm{jour}$, l'AUDC représente $40-50 \%$ du pool des acides biliaires [2]. En raison de leurs capacités détergentes, les acides biliaires plus hydrophobes sont toxiques pour les 
membranes cellulaires, induisant des lésions hépatocellulaires et un accroissement de la cholestase. L'AUDC exerce un effet stabilisateur de membrane réduisant la rupture des membranes riches en cholestérol [3].

- L'AUDC, mais non sa forme conjuguée taurodésoxycholate, diminue la toxicité des acides biliaires lipophiles pour la fonction de la chaîne de transport d'électrons d'une façon dépendante de la concentration (jusqu'à $100 \mu \mathrm{mol} / \mathrm{l}$ ) mais accroît la toxicité mitochondriale induite par les acides biliaires à des concentrations plus élevées [4].

- Interférence avec l'absorption d'acides biliaires plus toxiques dans l'iléon [2].

- L'AUDC est réabsorbé par l'épithélium biliaire sous forme protonée et est secrété à nouveau dans la bile (dérivation cholé-hépatique), produisant un effet hypercholérétique. Les protons absorbés aboutissent à la formation de bicarbonates qui sont secrétés et enrichissent la bile en cette composante [2].

- L'AUDC accroît le flux indépendant des sels biliaires $[1,2]$.

- L'AUDC peut stimuler l'expression de transporteurs à la surface canaliculaire des hépatocytes [5].

- Une réduction de l'expression des antigènes leucocytaires humains de classe I (HLA-I) à la surface des hépatocytes a été rapportée [6].

- L'AUDC inhibe également l'induction médiée par l'interféron $\gamma$ de l'expression des antigènes leucocytaires humains de classe II (HLA-II) par l'intermédiaire de la voie médiée par les récepteurs des glucocorticoïdes [7].

Les effets indésirables de l'AUDC sont très rares et transitoires, par exemple une diarrhée et des réactions cutanées plus probablement dues à des adjuvants du produit qu'à la substance active. L'AUDC doit être cependant évité ou administré avec prudence chez les patients présentant une affection obstructive des voies biliaires. Chez l'animal, après ligature du canalbiliaire principal, l'AUDC peut aggraver des infarctus biliaires [8]. L'AUDC peut accélérer la survenue d'une insuffisance hépatique chez un enfant atteint d'atrésie biliaire après l'échec d'une intervention de Kasai.

L'AUDC est l'agent le plus fréquemment administré chez les enfants atteints de cholestase chronique. Le bénéfice de l'administration à long terme de l'AUDC n'a pas été démontré de façon formelle dans plusieurs affections, par exemple la mucoviscidose. Dans d'autres cas, l'administration de ce produit améliore les paramètres fonctionnels hépatiques, mais non l'évolution finale, par exemple chez les patients atteints de cholangite sclérosante. Dans la CIFP, il paraît arrêter la progression de la maladie chez certains enfants et, associé à l'acide cholique, est le traitement de choix des patients présentant des erreurs innées du métabolisme des acides biliaires. Chez l'enfant atteint de syndrome d'Alagille ou d'atrésie biliaire, l'AUDC est principalement administré afin de réduire des symptômes tels le prurit.

\section{Mucoviscidose}

Au bout de 2 mois de traitement par l'AUDC, cet acide biliaire représente 25 à $42 \%$ du pool, aux dépens des acides cholique et chénodésoxycholique. Ces différences dépendent de la dose [9]. Ces études ont permis de conclure que la posologie recommandée de l'AUDC était supérieure ou égale à $20 \mathrm{mg} / \mathrm{kg} /$ jour [9].

L'AUDC stimule la capacité de sécrétion biliaire dans le foie des patients atteints de mucoviscidose, comme le montre la scintigraphie hépatobiliaire [10]. Plusieurs études pilotes ont montré que l'AUDC était responsable d'une amélioration de tests biochimiques, principalement une diminution du taux sérique des aminotransférases (ALAT et ASAT) et de la $\gamma$-glutamyl transférase (GGT) [11]. De plus, l'inflammation hépatique et/ou la prolifération des canaux biliaires ont été améliorées au bout de 2 ans de traitement par l'AUDC dans des biopsies de contrôle. L'administration de l'AUDC améliore également le statut nutritionnel chez l'adulte jeune atteint de mucoviscidose et, chez l'enfant, améliore le statut des acides gras essentiels et du rétinol [12-14]. L'AUDC n'influence cependant pas la stéatorrhée en raison de sa médiocre capacité à former des micelles [13]. Si l'AUDC modifiel'histoire naturelle del'hépatopathie chez les patients mucoviscidosiques, il doit encore être étudié dans le cadre d'une importante étude prospective randomisée. Des résultats encourageants ont été récemment rapportés concernant l'arrêt de la progression ou même la régression de lésions hépatiques échographiques sous l'effet de l'AUDC chez des patients atteints de mucoviscidose dans une étude à long terme [15].

\section{Nutrition parentérale totale}

L'absence d'apports oraux associée à une nutrition parentérale totale (NPT) aboutit à une stase biliaire liée à la stimulation insuffisante du flux biliaire dépendant et indépendant des acides biliaires. Dans un modèle de cholestase induite par une NPT, l'administration intraveineuse d'AUDC a amélioré le flux biliaire et a réduit la bilirubinémie [16]. Des résultats encourageants similaires ont été notés lors d'une étude pilote chez des enfants 
atteints de diarrhée intraitable et dépendant d'une NPT, mais tolérant l'AUDC à la posologie de $30 \mathrm{mg} / \mathrm{kg} /$ jour en 3 prises [17].

Chez des nourrissons de très petit poids de naissance présentant une cholestase associée à une NPT, l'AUDC à doses de $10-30 \mathrm{mg} / \mathrm{kg} / \mathrm{jour}$ a réduit la durée et l'intensité de la cholestase [18]. Chez la plupart de ces patients, des complications intestinales, dont des résections intestinales partielles, s'opposent à l'administration standard de l'AUDC. Par la suite, une dose quotidienne très fractionnée de cet agent peut être bien tolérée, évitant une diarrhée. La forme intraveineuse de l'AUDC n'est malheureusement pas commercialisée.

\section{Erreurs innées du métabolisme des acides biliaires}

Deux principales formes d'anomalies de la synthèse des acides biliaires sont responsables d'une cholestase chronique chez l'enfant: le déficit en 3-oxo- $\Delta 4$-stéroïde$5 \beta$-réductase et le déficit en $3 \beta$-hydrostéroïde- $\Delta 5$-oxyréductase/isomérase. L'hépatotoxicité est probablement due à l'accumulation de précurseurs d'acides biliaires et à l'insuffisance des acides biliaires primaires. Afin de réduire la synthèse de molécules toxiques, l'activité de la cholestérol-7 $\alpha$-hydro-oxylase, l'enzyme limitant le taux lors de la synthèse des acides biliaires endogènes, doit être impérativement inhibée.

La plupart des patients ne répondent que partiellement à un traitement par l'AUDC seul. Lassociation de l'acide cholique (250 mg/jour) est donc indiquée. L'administration des acides chénodésoxycholique et cholique a été également proposée chez ces patients. Ce dernier traitement peut se traduire par une amélioration plus rapide des lésions hépatiques [19].

\section{Cholestase intrahépatique familiale progressive}

L'AUDC est un traitement initial précieux pour les patients atteints de CIFP quand il est administré par voie orale à une dose de 20 à $30 \mathrm{mg} / \mathrm{kg} /$ jour. Des améliorations similaires ont été observées indépendamment du type de CIFP: des patients présentant un taux sérique de GGT normal (CIFP types 1 et 2) ou élevé (CIFP type 3 ) ont été améliorés de façon similaire. Chez les patients dont le taux de GGT était normal, une amélioration des paramètres de la cholestase a été observée dans $61 \%$ des cas, et dans $71 \%$ de ceux de CIFP de type 3 [20]. Lancienneté de la maladie avant l'instauration de l'AUDC pourrait influencer ces résultats. Un diagnostic précoce et une instauration rapide du traitement par AUDC pourraient davantage améliorer le résultat final. Chez certains patients, l'amélioration, même si elle est totale, n'est que transi- toire et la cholestase réapparaît quelques années après l'instauration de l'AUDC.

Une cholestase intrahépatique bénigne récidivante s'observe chezles patients présentant des mutations moins délétères de gènes responsables du type 1 ou 2 de la CIFP. Chez les patients présentant ce type de cholestase, le traitement par AUDC ne prévient pas les épisodes cholestatiques mais pourrait améliorer les symptômes (par exemple le prurit) [21].

\section{Cholangite sclérosante}

Une étude pilote initiale de l'AUDC chez des patients présentant une cholangite sclérosante bien constituée a montré une amélioration des paramètres fonctionnels hépatiques. Par la suite, une différence statistiquement significative des taux sériques d'ALAT/ASAT/GGT a été observée entre les patients du groupe AUDC et ceux du groupe placebo atteints de cholangite sclérosante au cours d'une étude comportant 3 ans de suivi [22]. Une amélioration du prurit et de l'asthénie a été également observée, mais la différence n'a pas été significative. Malheureusement, des résultats plus récents d'une étude en double insu et randomisée comparant l'AUDC (13-15 mg/kg/ jour) à un placebo n'ont révélé aucune différence en ce qui conerne les échecs thérapeutiques (progression de la maladie, décès ou transplantation hépatique). Cette étude a confirmé une amélioration des paramètres fonctionnels hépatiques, mais a conclu que l'AUDC n'offrait pas de bénéfice clinique aux patients atteints de cholangite sclérosante [23]. De ce fait, l'AUDC à faible dose ne modifie apparemment pas la progression de cette maladie. Une étude très récemment publiée a montré que, au contraire, des doses élevées d'AUDC avaient été associées à une amélioration de la survie et à une tendance à une stabilité ou une amélioration du stade histologique dans un groupe relativement restreint de patients atteints de cholangite sclérosante [24].

Malgré ces résultats, de nouveaux essais pourraient être proposés, utilisant des doses élevées d'AUDC très précocement dans l'évolution de la cholangite sclérosante et particulièrement chez des patients présentant des atteintes des petits canaux. Quand la maladie affecte les voies biliaires principales, comme dans la plupart des cas, la perturbation du flux biliaire peut probablement contribuer de façon irréversible à l'aggravation de l'hépatopathie. La plus grande expérience de l'utilisation de l'AUDC dans des affections cholestatiques chroniques a été obtenue chez des patients adultes atteints de cirrhose biliaire primaire, forme sévère de cholangiopathie intrahépatique [25]. Différentes équipes ont présenté des résultats 
divergents obtenus avec l'AUDC, partiellement expliqués par les anciennetés différentes de la maladie au début du traitement. La dose d'AUDC administrée a également paru jouer un rôle dans l'influence de ce médicament sur l'évolution finale. Cette importante expérience doit être prise en compte pour la conception de nouveaux essais chez des patients atteints de cholangite sclérosante.

\section{Atrésie des voies biliaires}

Une étude préliminaire précoce de l'AUDC après portoentérostomie hépatique a montré que cet agent était efficace chez les patients dont le drainage biliaire postopératoire était satisfaisant, entraînant des taux plus faibles d'acide biliaire total et un meilleur gain de poids [26] L'administration à long terme de l'AUDC a significativement amélioré l'évolution finale après une portoentérostomie de Kasai. L'ictère a disparu chez un plus grand nombre de patients, qui ont survécu sans transplantation hépatique. Chez les enfants sans rétablissement d'un flux biliaire satisfaisant après portoentérostomie, l'administration de l'AUDC peut aboutir à une progession plus rapide des lésions hépatiques et, par la suite, accélérer la survenue d'une insuffisance hépatique [27].

\section{Syndrome d'Alagille}

Peu de publications sont disponibles quant à l'utilisation de l'AUDC chez des patients atteints de syndrome d'Alagille. Une amélioration de l'ictère et du prurit a été observée, ainsi qu'une diminution des taux des lipides circulants et une réduction des xanthomes [28]. Aucune étude contrôlée n'est disponible mais l'AUDC est largement prescrit à ces patients. L'influence de cet acide biliaire sur l'histoire naturelle de la maladie est encore inconnue.

\section{Rifampicine}

La rifampicine diminue la concentration des acides biliaires dans les hépatocytes en entrant en compétition avec leur captation. De plus, cet antibiotique induit les enzymes microsomiales qui favorisent la $6 \alpha$-hydroxylation et la $6 \alpha$-glucuronisation consécutive des acides biliaires toxiques.

L'expérience clinique initiale de l'utilisation de cet antibiotique dans le traitement du prurit a été obtenue chez des adultes atteints de cirrhose biliaire primitive, chez la plupart desquels un soulagement significatif a été observé [29]. Chez des enfants atteints de cholestase chronique, une étude croisée en double insu a montré l'efficacité de la rifampicine dans le soulagement du prurit sur des pé- riodes allant jusqu'à 6 mois [30]. Deux études menées par la suite chez des patients pédiatriques souffrant de prurit réfractaire traités par la rifampicine ont confirmé ces résultats initiaux $[31,32]$. Aucune amélioration des caractéristiques biochimiques de la cholestase n'a été enregistrée chez la plupart des enfants traités atteints de cholestase chronique de différentes causes.

La posologie recommandée de la rifampicine est de 10$20 \mathrm{mg} / \mathrm{kg} / \mathrm{jour}$ en deux prises. Peu d'effets indésirables ont été rapportés. La rifampicine est cependant un inducteur des enzymes microsomiales. L'activité de plusieurs des isoenzymes du cytochrome P450 est accrue par l'administration de la rifampicine et, de ce fait, le taux du métabolisme de plusieurs médicaments peut être accéléré.

Des auteurs ont suggéré que l'AUDC et la rifampicine pouvaient exercer des effets complémentaires afin de justifier leur association dans le traitement de patients atteints de maladies cholestatiques [33].

\section{Résines échangeuses d'ions non absorbables}

Ces molécules ne sont pas absorbables par les voies digestives, libèrent des chlorures et fixent les acides biliaires dans la lumière de l'intestin, ce qui diminue leur recirculation entérohépatique. Ces résines accroissent l'excrétion du cholestérol en amplifiant la conversion en acides biliaires. Ces agents peuvent diminuer d'environ $40 \%$ le pool des acides biliaires. L'acide biliaire trihydroxy se dissocie rapidement de ces molécules et peut être absorbé dans l'iléon.

La cholestyramine (granulés aromatisés à l'orange) est utilisée depuis plusieurs décennies et s'est avérée efficace et bien tolérée. Sa palatabilité est cependant médiocre, ce qui limite son acceptabilité. De ce fait, l'observance est erratique dans le meilleur des cas. En cas de mise en place d'une sonde nasogastrique pour nutrition entérale, l'administration de la cholestyramine par cette voie s'est avérée bien tolérée et efficace, entraînant la diminution de la cholestérolémie et la régression du prurit (fig. 1).

Chez l'enfant, la cholestyramine peut être instaurée à une dose de 2-4 g deux fois par jour augmentée ensuite à 8-16 g/jour en 2 ou 3 prises. La cholestyramine fixe d'autres acides ou des substances négativement chargées, ce qui explique la plupart de ses interactions avec de nombreux médicaments.

Le colestipol sous forme de granulés ou de comprimés n’a pas été fréquemment utilisé chez l'enfant. Ses indications et précautions d'emploi sont similaires à celles décrites pour la cholestyramine. 


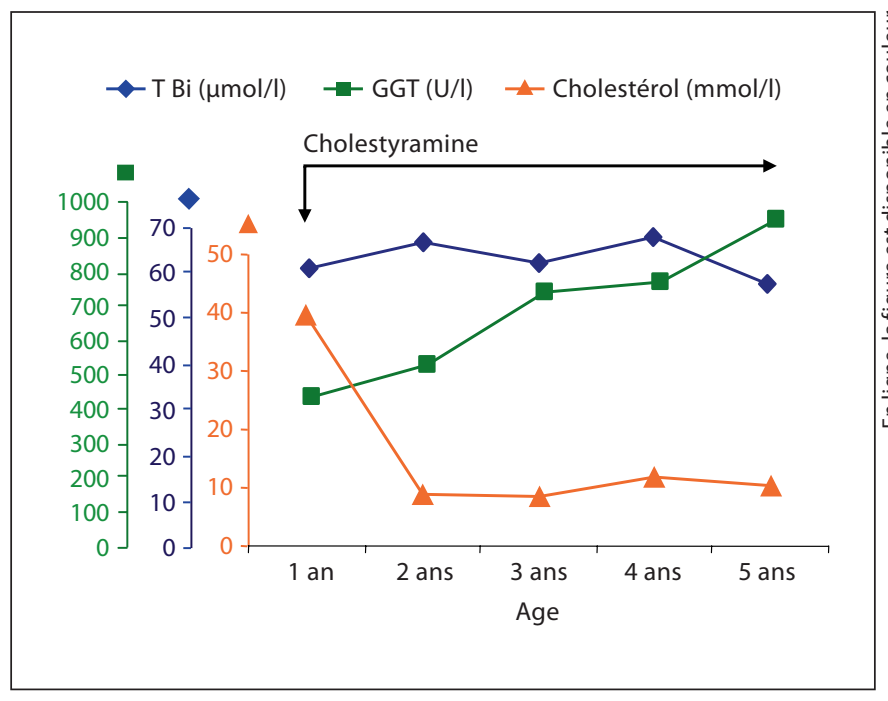

Fig. 1. Chez ce garçon atteint de syndrome d'Alagille, la cholestyramine a été administrée par sonde nasogastrique, indiquée pour une nutrition entérale. Une très forte diminution de la cholestérolémie avec réduction du volume des xanthomes a été observée. Le prurit s'est amélioré avec l'association de la rifampicine au traitement. Aucune modification des paramètres biochimiques de cholestase n'a été enregistrée.

Le chlorhydrate de colésévélam (sous forme d'hydrogel ou de comprimé non absorbable) est probablement le mieux toléré de ces produits mais n'est malheureusement pas disponible partout [34]. Cet agent présente une meilleure spécificité, une plus grande affinité et une capacité plus élevée de liaison des acides biliaires comparativement aux 2 autres résines échangeuses d'ions non absorbables mentionnées plus haut.

Les résines échangeuses d'ions non absorbables peuvent interférer avec l'absorption intestinale d'autres médicaments, par exemple l'AUDC. Ce problème particulier doit être pris en compte lors du traitement d'un patient atteint de cholestase chronique. De plus, le moment d'administration doit être bien établi afin de permettre une capture maximale des acides biliaires et d'éviter une interaction avec d'autres médicaments, dont les vitamines liposolubles. Les autres médicaments doivent être administrés 1 ou 4-6 heures après la cholestyramine.

Les principaux effets indésirables sont une constipation et une acidose hyperchlorémique due à l'importante quantité de chlorures libérés dans la lumière gastro-intestinale et absorbés à la place des bicarbonates. Cette dernière complication peut être particulièrement sévère chez des patients atteints de syndrome d'Alagille, qui présentent fréquemment des antécédents de troubles rénaux.

\section{Antagonistes des opiacés}

La naloxone et le nalméfène sont les deux premiers antagonistes des opiacés évalués chez des adultes atteints de cirrhose biliaire primaire, afin de tenter de soulager le prurit $[35,36]$. Ladministration de ces substances reposait sur le rôle éventuel du système nerveux central dans le prurit observé chez les patients atteints de cholestase. Des substances de la classe des enképhalines (opioïdes endogènes) pourraient être des médiateurs potentiels dans l'intervention du système nerveux.

Un essai en simple insu contrôlé contre placebo de la naloxone a démontré que ce médicament réduisait l'activité de grattage chez des patients cholestatiques. Les principaux problèmes observés avec ce produit ont été la nécessité de perfusions intraveineuses en raison de sa très courte demi-vie et un éventuel syndrome de sevrage des opiacés à la fin de la perfusion [35].

Le nalméfène peut être administré par voie orale (mais cette forme n'est pas disponible partout). Une amélioration de la perception du prurit a été rapportée chez la plupart des patients traités, en raison de la plus longue durée d'action de ce produit comparativement à la naloxone. Ce médicament peut être associé à des manifestations sévères de sevrage, qui dépendent apparemment de la dose administrée. Une importante exacerbation du prurit a été également rapportée à la suite de son arrêt brutal [36].

La naltrexone peut également être administrée par voie orale. Un essai randomisé de la naltrexone comparativement à un placebo a montré une diminution significative du prurit diurne et nocturne. Un syndrome de sevrage des opiacés a été observé chez $50 \%$ des patients en recevant [37].

Aucune série sur l'utilisation d'antagonistes d'opiacés chez des enfants atteints de cholestase chronique n'a été publiée. De ce fait, il est impossible de conclure quant à l'indication de ces produits dans la population pédiatrique. Sur la base de l'expérience acquise chez l'adulte, ces médicaments sont d'administration difficile et il est délicat d'éviter des effets secondaires à leur arrêt.

\section{Acides gras $\omega-3$}

Une hépatopathie associée à une NPT chez des enfants présentant une insuffisance intestinale peut aboutir à une cirrhose et une insuffisance hépatique. Chez certains patients, quand l'AUDC était absorbé, une amélioration des paramètres fonctionnels hépatiques a été notée. Les résultats les plus spectaculaires ont été obte- 
nus en remplaçant les huiles de soja par des huiles de poisson dans les émulsions lipidiques. Les enfants recevant une solution enrichie en acides gras $\omega-3$ ont présenté une amélioration très importante de leurs lésions hépatiques [38].

Récemment, un garçon âgé de 4 ans suivi dans notre institution a fait l'objet d'une résection de la plus grande partie de l'intestin grêle en raison d'un volvulus. Cet enfant a présenté une hépatopathie sévère sous NPT et a été inscrit sur la liste d'attente pour transplantation hépatique. Une administration d'huile de poisson (omégaven) a été instaurée et, 3 mois plus tard, l'enfant a été rayé de la liste d'attente. Six mois plus tard, il présentait des taux sériques normaux de bilirubine conjuguée [V. Marchand, communication personnelle].

\section{$\mathrm{N}$-acétyl cystéine}

Dans la population recevant une NPT à long terme, la survenue d'une hépatopathie est la conséquence de divers facteurs. Le stress oxydatif paraît jouer un rôle majeur dans l'étiologie et la persistance ainsi que dans l'aggravation d'une cholestase. Les concentrations cellulaires de glutathion sont une défense essentielle contre ce processus nocif. La cystéine est un important précurseur du glutathion intracellulaire, mais les solutions d'acides aminés actuellement utilisées en contiennent de faibles taux.

La N-acétyl cystéine (NAC) peut être administrée à titre de précurseur de la cystéine. Une normalisation ou une forte diminution de la bilirubinémie conjuguée a été enregistrée après l'ajout de la NAC à une solution de nutrition parentérale à la dose de $70-135 \mathrm{mg} / \mathrm{kg} /$ jour [39]. Lamélioration de la cholestase hépatique a été associée à une normalisation du glutathion dans les érythrocytes, suggérant que la concentration cellulaire de cette molécule antioxydante avait joué un rôle majeur dans la régression des lésions hépatiques.

\section{Interventions chirurgicales}

\section{Dérivation biliaire et exclusion iléale}

Voici plus de 30 ans, Alagille et Odièvre, à l'Hôpital Bicêtre en France, ont décrit l'efficacité du drainage biliaire par cholécystostomie chez des patients atteints de CIFP et de syndrome d'Alagille. Malheureusement, en raison de complications cutanées locales autour du drain, ce type de drainage biliaire externe n'a pu être maintenu pendant une longue durée [40].

Traitements de la cholestase

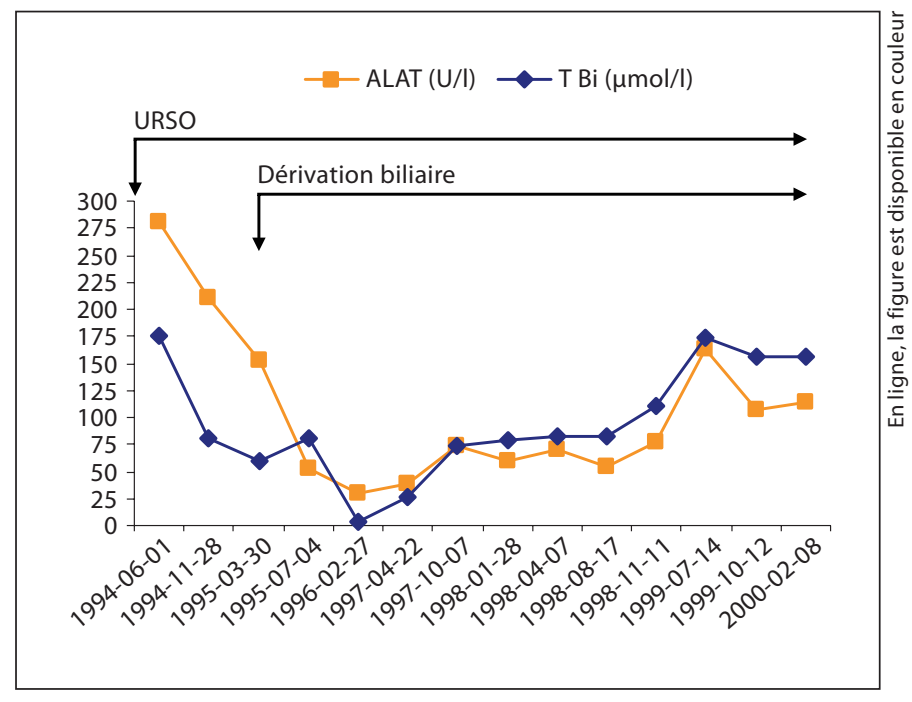

Fig. 2. Une dérivation biliaire partielle avait été effectuée chez cette fille atteinte de CIFP (GGT basse). Une normalisation quasitotale des paramètres biochimiques hépatiques avait été enregistrée. Une récidive des signes et symptômes de cholestase a été observée 2-3 ans après l'intervention chirurgicale. Une scintigraphie hépatobiliaire a montré que le flux de bile était presque exclusivement dirigé vers la poche pour stomie. Ce cas a montré que l'hépatopathie avait progressé malgré une dérivation biliaire et un traitement par AUDC.

Dans les années 1980, une technique de dérivation biliaire partielle a été développée, utilisant l'interposition de 10 à $15 \mathrm{~cm}$ de jéjunum entre la vésicule biliaire et la peau. Cette intervention était permanente et permettait la dérivation partielle du flux biliaire vers une poche externe pour stomie pendant plusieurs années. Une amélioration clinique et biochimique a été rapportée chez des patients atteints de syndrome d'Alagille [41]. Chez des enfants présentant une CIFP, la dérivation biliaire partielle a entraîné une amélioration de la croissance, un soulagement du prurit et une correction des anomalies biochimiques de la cholestase $[42,43]$. Dans certains cas, l'amélioration a été spectaculaire mais transitoire, suivie d'une récidive de la cholestase et de la nécessité d'une transplantation hépatique 4 à 5 ans après la dérivation biliaire initiale (observations personnelles) (fig. 2). Dans d'autres cas, l'amélioration complète n'a été obtenue qu'avec l'administration d'AUDC à titre de traitement coadjuvant.

Une variante technique a été décrite par la suite, dans laquelle l'appendice vermiforme a été utilisé à titre de conduit entre la vésicule biliaire et la peau, avec de très bons résultats [44].

Ann Nestlé [Fr] 2008;66:127-136 
Une publication récente a décrit une autre façon d'obtenir une dérivation partielle du flux biliaire. Chez trois patients atteints de syndrome d'Alagille, 15\% de l'iléon terminal ont été exclus au moyen d'une division par agrafes et d'une anastomose iléo-cæcale. La justification de l'intervention était d'exclure le site d'absorption maximale des acides biliaires dans l'intestin. Une diminution de la cholestérolémie, une amélioration du prurit et une réduction de la taille des xanthomes ont été observées [45].

Toutes ces interventions chirurgicales devraient être évitées chez les patients déjà atteints d'une cirrhose. D'importants saignements de néovarices aux sites de l'anastomose sont des complications très fréquentes. De plus, les bénéfices de ces interventions ne sont pas démontrés chez les patients atteints d'hépatopathie avancée.

\section{Portoentérostomie}

L'intervention chirurgicale décrite par Kasai dans les années 1950 est devenue la première et seule à même de restaurer le flux biliaire chez les patients atteints d'atrésie des voies biliaires

Une normalisation de la bilirubinémie dans les 3 mois suivant une portoentérostomie indique un bon pronostic à long terme. Cette situation s'observe chez environ 50 $60 \%$ des nourrissons opérés, avec une meilleure évolution quand l'intervention a été réalisée précocement [46]. D’autres facteurs présents au moment de la portoentérostomie peuvent modifier le résultat final, par exemple un certain degré de fibrose hépatique et les caractéristiques du résidu extrahépatique. Comparativement à l'atrésie des voies biliaires sans malformation, le pronostic del'intervention de Kasai chez des patients présentant un syndrome de malformation polysplénique associé à une atrésie des voies biliaires est plus mauvais dans certains centres et similaire dans d'autres [47]. Au cours de la période postopératoire, la survenue d'une cholangite peut également assombrir le pronostic.

En raison de la présence d'infiltrats inflammatoires dans le foie de ces patients, des auteurs ont suggéré que l'administration d'anti-inflammatoires pourrait arrêter la progression de la maladie. Malheureusement, plusieurs publications récentes d'études pilotes et randomisées en double insu ont montré que l'administration de corticoïdes à doses élevées après une intervention de Kasai n’améliorait pas la survie du foie natif $[48,49]$.

Le taux de survie à 10 ans est de $30-54 \%$ dans différents centres au plan international, de $25 \%$ à 20 ans et de
$10 \%$ à 30 ans [50-53]. Tous les patients présentent une cirrhose, avec les complications associées d'une hypertension portale: saignements digestifs, splénomégalie et atteintes hépatopulmonaires.

La transplantation hépatique a amélioré la survie globale des enfants atteints d'atrésie biliaire après un échec de l'intervention de Kasai. Actuellement, le succès de ce traitement est responsable d'un taux de survie de $90 \%$ [47].

\section{Perspectives}

Dans l'avenir, deux traitements pourraient permettre d'obtenir la guérison définitive de certaines maladies héréditaires responsables d'une cholestase chronique: la thérapie génique et/ou la transplantation d'hépatocytes. Dans le cas de la thérapie génique, après des années initiales d'espoir, les résultats décevants de la plupart des essais et des effets indésirables graves ont ralenti sa progression et la poursuite de son développement. Dans le cas du foie, on ne sait pas combien de cellules devraient exprimer un gène spécifique pour que la survenue des signes et symptômes d'une maladie particulière soit évitée et que sa progression ralentisse. De nombreux problèmes doivent être résolus avant de pouvoir même concevoir un essai concernant une hépatopathie cholestatique.

Une transplantation d'hépatocytes a déjà été tentée chez certains patients. Une correction partielle de l'atteinte hépatique héritée a été observée dans des modèles animaux. Les hépatocytes normaux ont un avantage pour la repopulation du foie par rapport à des cellules porteuses d'un gène muté et peut-être létal. Une correction de la CIFP de type 3 dans un modèle murin a été décrite et est demeurée stable pendant une longue durée [54]. Ce traitement se heurte à la disponibilité d'hépatocytes normaux, à la nécessité d'un traitement immunosuppresseur et à la question du devenir des cellules mutées restantes. Le risque d'apparition de tumeurs au cours du suivi à long terme est une préoccupation considérable.

L'administration des acides gras $\omega-3$ dans des cholestases de différentes origines doit être explorée. Une carence en acide gras essentiels a été rapportée chez les patients atteints d'une cholestase de différentes étiologies, par exemple syndrome d'Alagille et mucoviscidose. Cette caractéristique est très probablement une conséquence d'une stéatorrhée sévère. Des voies extra-intestinales d'administration ont été proposées, mais sans grand succès. Une administration intraveineuse périodique pourrait être indiquée dans certains cas. 
Un stress oxydatif est presque constamment associé à la cholestase. On peut supposer que, chez ces enfants, la rétention de composants de la bile, la malabsorption responsable d'une malnutrition et une carence en acides gras essentiels et en oligoéléments aboutissent à des anomalies de différents mécanismes cellulaires protecteurs. Dans ce dernier cas, le déficit en glutathion peut être inclus. Ladministration de NAC pourrait être très utile dans plusieurs processus cholestatiques à titre de traitement adjuvant.

Des essais à venir devront évaluer le bénéfice potentiel des acides gras $\omega$-3 et de la NAC chez des enfants atteints de cholestase chronique. Ces deux traitements exercent très peu d'effets indésirables, étant bien tolérés chez l'enfant.

Un grand nombre des médicaments décrits plus haut pourraient être associés dans le traitement de la cholestase chronique afin de diminuer les lésions hépatiques et/ou de soulager des symptômes nocifs liés à ces affections. Des agents dotés de mécanismes d'action différents peuvent être associés, avec un soutien nutritionnel adéquat, en individualisant le traitement en fonction de l'étiologie de la cholestase et de l'état clinique actuel de l'enfant considéré.

\section{Bibliographie}

1 Heuman DM: Hepatoprotective properties of ursodeoxycholic acid. Gastroenterology 1993;104:1865-1870.

2 Beuers U, Boyer JL, Paumgartner G: Ursodeoxycholic acid in cholestasis: potential mechanisms of action and therapeutic applications. Hepatology 1998;28:1449-1453.

3 Heuman DM, Bajaj R: Ursodeoxycholate conjugates protect against disruption of cholesterol-rich membranes by bile salts. Gastroenterology 1994;106:1333-1341.

4 Krahenbuhl S, Fischer S, Talos C, Reichen J: Ursodeoxycholate protects oxidative mitochondrial metabolism from bile acid toxicity: dose-response study in isolated rat liver mitochondria. Hepatology 1994;20:15951601.

5 Fickert P, Zollner G, Fuchsbichler A, et al: Effects of ursodeoxycholic and cholic acid feeding on hepatocellular transporter expression in mouse liver. Gastroenterology 2001;121:170-183.

-6 Terasaki S, Nakanuma Y, Ogino H, et al: Hepatocellular and biliary expression of HLA antigens in primary biliary cirrhosis before and after ursodeoxycholic acid therapy. Am J Gastroenterol 1991;86:1194-1199.

7 Tanaka H, Makino Y, Miura T, et al: Ligandindependent activation of the glucocorticoid receptor by ursodeoxycholic acid. Repression of IFN-gamma-induced MHC class II gene expression via a glucocorticoid receptor-dependent pathway. J Immunol 1996; 156:1601-1608.

-8 Fickert P, Zollner G, Fuchsbichler A, et al: Ursodeoxycholic acid aggravates bile infarcts in bile duct-ligated and Mdr2 knockout mice via disruption of cholangioles. Gas troenterology 2002;123:1238-1251.

-9 Colombo C, Crosignani A, Assaisso M, et al: Ursodeoxycholic acid therapy in cystic fibrosis-associated liver disease: a dose-response study. Hepatology 1992;16:924-930.
10 Colombo C, Castellani MR, Balistreri WF, et al: Scintigraphic documentation of an improvement in hepatobiliary excretory function after treatment with ursodeoxycholic acid in patients with cystic fibrosis and associated liver disease. Hepatology 1992;15: 677-684.

11 Colombo C, Setchell KD, Podda M, et al: Effects of ursodeoxycholic acid therapy for liver disease associated with cystic fibrosis. J Pediatr 1990;117:482-489.

12 Lindblad A, Glaumann H, Strandvik B: A two-year prospective study of the effect of ursodeoxycholic acid on urinary bile acid excretion and liver morphology in cystic fibrosis-associated liver disease. Hepatology 1998;27:166-174.

13 Cotting J, Lentze MJ, Reichen J: Effects of ursodeoxycholic acid treatment on nutrition and liver function in patients with cystic fibrosis and longstanding cholestasis. Gut 1990;31:918-921.

14 Lepage G, Paradis K, Lacaille F, et al: Ursodeoxycholic acid improves the hepatic metabolism of essential fatty acids and retinol in children with cystic fibrosis. J Pediatr 1997;130:52-58.

15 Nousia-Arvanitakis S, Fotoulaki M, Economou H, et al: Long-term prospective study of the effect of ursodeoxycholic acid on cystic fibrosis-related liver disease. J Clin Gastroenterol 2001;32:324-328.

16 Duerksen DR, Van Aerde JE, Gramlich L, et al: Intravenous ursodeoxycholic acid reduces cholestasis in parenterally fed newborn piglets. Gastroenterology 1996;111:11111117.

17 Spagnuolo MI, Iorio R, Vegnente A, Guarino A: Ursodeoxycholic acid for treatment of cholestasis in children on long-term total parenteral nutrition: a pilot study. Gastroenterology 1996;111:716-719.
8 Chen CY, Tsao PN, Chen HL, et al: Ursodeoxycholic acid (UDCA) therapy in very-lowbirth-weight infants with parenteral nutrition-associated cholestasis. J Pediatr 2004; 145:317-321.

19 Clayton PT, Mills KA, Johnson AW, et al: Delta 4-3-oxosteroid 5 beta-reductase deficiency: failure of ursodeoxycholic acid treatment and response to chenodeoxycholic acid plus cholic acid. Gut 1996;38:623-628.

-20 Jacquemin E, Hermans D, Myara A, et al: Ursodeoxycholic acid therapy in pediatric patients with progressive familial intrahepatic cholestasis. Hepatology 1997;25:519-523.

21 Crosignani A, Podda M, Bertolini E, et al: Failure of ursodeoxycholic acid to prevent a cholestatic episode in a patient with benign recurrent intrahepatic cholestasis: a study of bile acid metabolism. Hepatology 1991;13: 1076-1083.

22 Stiehl A, Walker S, Stiehl L, et al: Effect of ursodeoxycholic acid on liver and bile duct disease in primary sclerosing cholangitis. A 3 -year pilot study with a placebo-controlled study period. J Hepatol 1994;20:57-64.

23 Lindor KD: Ursodiol for primary sclerosing cholangitis. Mayo Primary Sclerosing Cholangitis-Ursodeoxycholic Acid Study Group. N Engl J Med 1997;336:691-695.

24 Cullen SN, Rust C, Fleming K, et al: High dose ursodeoxycholic acid for the treatment of primary sclerosing cholangitis is safe and effective. J Hepatol 2008;48:792-800.

25 Corpechot C, Carrat F, Bahr A, et al: The effect of ursodeoxycholic acid therapy on the natural course of primary biliary cirrhosis. Gastroenterology 2005;128:297-303.

26 Nittono H, Tokita A, Hayashi M, et al: Ursodeoxycholic acid in biliary atresia. Lancet 1988;1:528.

-27 Stringer MD, Davison SM, Rajwal SR, McClean P: Kasai portoenterostomy: 12-year experience with a novel adjuvant therapy regimen. J Pediatr Surg 2007;42:1324-1328. 
28 Krawinkel MB, Santer R, Oldigs HD: Ursodesoxycholic acid: effect on xanthomas in Alagille-Watson syndrome. J Pediatr Gastroenterol Nutr 1994;19:476-477.

-29 Bachs L, Parés A, Elena M, et al: Comparison of rifampicin with phenobarbitone for treatment of pruritus in biliary cirrhosis. Lancet 1989;1:574-576.

>30 Cynamon HA, Andres JM, Iafrate RP: Rifampin relieves pruritus in children with cholestatic liver disease. Gastroenterology 1990;98:1013-1016.

-31 Gregorio GV, Ball CS, Mowat AP, Mieli-Vergani G: Effect of rifampicin in the treatment of pruritus in hepatic cholestasis. Arch Dis Child 1993;69:141-143.

-32 Yerushalmi B, Sokol RJ, Narkewicz MR, et al: Use of rifampin for severe pruritus in children with chronic cholestasis. J Pediatr Gastroenterol Nutr 1999;29:442-447.

-33 Marschall HU, Wagner M, Zollner G, et al: Complementary stimulation of hepatobiliary transport and detoxification systems by rifampicin and ursodeoxycholic acid in humans. Gastroenterology 2005;129:476-485.

>34 Aldridge MA, Ito MK: Colesevelam hydrochloride: a novel bile acid-binding resin. Ann Pharmacother 2001;35:898-907.

>35 Bergasa NV, Talbot TL, Alling DW, et al: A controlled trial of naloxone infusions for the pruritus of chronic cholestasis. Gastroenterology 1992;102:544-549.

>36 Bergasa NV, Schmitt JM, Talbot TL, et al: Open-label trial of oral nalmefene therapy for the pruritus of cholestasis. Hepatology 1998;27:679-684.

-37 Wolfhagen FH, Sternieri E, Hop WC, et al: Oral naltrexone treatment for cholestatic pruritus: a double-blind, placebo-controlled study. Gastroenterology 1997;113:12641269.
38 Gura KM, Duggan CP, Collier SB, et al: Reversal of parenteral nutrition-associated liver disease in two infants with short bowel syndrome using parenteral fish oil: implications for future management. Pediatrics 2006;118:e197-e201.

39 Mager DR, Marcon M, Wales P, Pencharz PB: Use of $\mathrm{N}$-acetyl cysteine for the treatment of parenteral nutrition-induced liver disease in children receiving home parenteral nutrition. J Pediatr Gastroenterol Nutr 2008;46: 220-223.

40 Alagille D, Odievre M: Les cholestases de l'enfant. Maladies du foie et des voies biliaires chez l'enfant. Paris, Flammarion, 1978.

41 Emerick KM, Whitington PF: Partial external biliary diversion for intractable pruritus and xanthomas in Alagille syndrome. Hepatology 2002;35:1501-1506.

42 Melter M, Rodeck B, Kardorff R, et al: Progressive familial intrahepatic cholestasis: partial biliary diversion normalizes serum lipids and improves growth in noncirrhotic patients. Am J Gastroenterol 2000;95:35223528.

43 Kurbegov AC, Setchell KD, Haas JE, et al: Biliary diversion for progressive familial intrahepatic cholestasis: improved liver morphology and bile acid profile. Gastroenterology 2003;125:1227-1234.

44 Gauderer MW, Boyle JT: Cholecystoappendicostomy in a child with Alagille syndrome. J Pediatr Surg 1997;32:166-167.

45 Modi BP, Suh MY, Jonas MM, et al: Ileal exclusion for refractory symptomatic cholestasis in Alagille syndrome. J Pediatr Surg 2007; 42:800-805.
46 Ohhama Y, Shinkai M, Fujita S, et al: Early prediction of long-term survival and the timing of liver transplantation after the Kasai operation. J Pediatr Surg 2000;35:10311034.

47 Chardot C, Serinet MO: Prognosis of biliary atresia: what can be further improved? J Pediatr 2006;148:432-435

48 Davenport M, Stringer MD, Tizzard SA, et al: Randomized, double-blind, placebo-controlled trial of corticosteroids after Kasai portoenterostomy for biliary atresia. Hepatology 2007;46:1821-1827.

49 Petersen C, Harder D, Melter M, et al: Postoperative high-dose steroids do not improve mid-term survival with native liver in biliary atresia. Am J Gastroenterol 2008;103:712719.

50 McKiernan PJ, Baker AJ, Kelly DA: The frequency and outcome of biliary atresia in the UK and Ireland. Lancet 2000;355:25-29.

51 Chardot C, Carton M, Spire-Bendelac N, et al: Prognosis of biliary atresia in the era of liver transplantation: French national study from 1986 to 1996. Hepatology 1999;30:606611.

52 Shneider BL, Brown MB, Haber B, et al: A multicenter study of the outcome of biliary atresia in the United States, 1997 to 2000. J Pediatr 2006;148:467-474.

-53 Schreiber RA, Barker CC, Roberts EA, et al: Canadian Pediatric Hepatology Research Group. Biliary atresia: the Canadian experience. J Pediatr 2007;151:659-665.

54 De Vree JM, Ottenhoff R, Bosma PJ, et al: Correction of liver disease by hepatocyte transplantation in a mouse model of progressive familial intrahepatic cholestasis. Gastroenterology 2000;119:1720-1730. 